Reprod. Nutr. Dévelop., 1986, 26 (1 B), 279-280.

\title{
Activités alimentaires et méryciques comparées des bovins, des caprins et des ovins
}

\author{
J. P. DULPHY, Brigitte CARLE
}

Laboratoire des Aliments,

I.N.R.A. Theix, 63122 Ceyrat, France.

Summary. The feeding and ruminating behaviour of lactating dairy cows, dry goats, dry ewes and wethers offered timothy hay or grass silage ad libitum have been compared. The main results are given in table 1 .

L'étude comparée des activités alimentaires et méryciques peut contribuer à expliquer certaines différentes entre les espèces de ruminants dans les quantités ingérées (Dulphy, Michalet-Doreau et Demarquilly, 1984). Jointes à des études sur la vitesse de transit, elles peuvent aussi expliquer des différences de digestibilité (Carle et Dulphy, 1980). Nous avons donc poursuivi ce travail.

Matériel et méthodes. Les mesures ont concerné deux fourrages (un foin tardif de fléole et un ensilage d'herbe jeune de prairie naturelle) qui ont été, chacun, distribué simultanément à 4 catégories d'animaux : béliers castrés de race Texel de 27 et 42 mois $(60-76 \mathrm{~kg})$, brebis de race Limousine taries de $43-50$ mois $(52-55 \mathrm{~kg})$, chèvres de race Alpine taries de $69-72$ mois (48 kg), vahes de race Holstein de 63 et 65 mois produisant 15 et $12 \mathrm{~kg}$ de lait $(546-560 \mathrm{~kg})$. Tous ces animaux ont été maintenus attachés individuellement en case à métabolisme. Le nombre retenu pour les mesures figure dans le tableau 1 . Les conditions de mesure sont celles données par Carle et Dulphy (1980) : deux distributions de fourrage par jour, refus de $10 \%$ environ, deux semaines d'adaptation et 6 jours de mesures de la digestibilité et des activités alimentaires.

Résultats et discussion. Les brebis ont ingéré moins (- $10 \%$ environ) que les béliers et pendant plus de temps ; elles ont ruminé pendant une durée comparable et ont eu une durée unitaire de rumination proche (tabl. 1). Les durées des grands repas ont été également comparables, ainsi que les temps de latence après ces grands repas, mais les brebis ont fait plus de repas que les béliers.

Les chèvres ont ingéré des quantités comparables aux brebis; elles ont ingéré le foin plus vite, mais l'ensilage à la même vitesse. Elles ont ruminé moins longtemps et leur temps de latence après les grands repas a été plus long.

Les vaches ont eu une durée d'ingestion, ainsi qu'un niveau d'ingestion par $\mathrm{kg}$ de poids métabolique, beaucoup plus élevés que les petits ruminants. La quantité de fourrage qu'elles ont mastiquée a été de $12,2 \mathrm{~g}$ de MS par minute, contre 1,8 pour les béliers et 1,5 pour les brebis et les chèvres. Malgré cela elles ont mieux digéré les deux fourrages, probablement à cause d'une durée de transit plus longue des fourrages : $75 \mathrm{~h}$ pour des particules colorées indigestibles, contre 55 heures seulement pour les béliers. 
TABL. 1. - Quantités de matière sèche ingérées et comportement alimentaire et mérycique des animaux ( \pm écart-type).

\begin{tabular}{|c|c|c|c|c|c|c|c|c|}
\hline \multirow[b]{2}{*}{$\begin{array}{l}\text { Type d'animal } \\
\text { (n) }\end{array}$} & \multicolumn{4}{|c|}{ Foin de fléole } & \multicolumn{4}{|c|}{ Ensilage de prairie naturelle } \\
\hline & $\begin{array}{l}V L \\
(6)\end{array}$ & $\begin{array}{l}\mathrm{CH} \\
\text { (5) }\end{array}$ & $\begin{array}{l}\mathrm{BE} \\
(5)\end{array}$ & $\begin{array}{l}B R \\
\text { (4) }\end{array}$ & $\begin{array}{l}V L \\
(6)\end{array}$ & $\begin{array}{l}\mathrm{CH} \\
(3)\end{array}$ & $\begin{array}{l}\mathrm{BE} \\
(6)\end{array}$ & $\begin{array}{l}B R \\
\text { (4) }\end{array}$ \\
\hline $\begin{array}{l}\text { Digestibilité } \\
\text { de la matière organique }\end{array}$ & $\begin{array}{l}63,3 a \\
(1,6)\end{array}$ & $\begin{array}{l}57,9 b \\
(1,8)\end{array}$ & $\begin{array}{l}55,6 b \\
(2,6)\end{array}$ & $\begin{array}{l}56,7 b \\
(2,1)\end{array}$ & $\begin{array}{l}80,5 a \\
(1,5)\end{array}$ & $\begin{array}{l}73,9 \mathrm{~b} \\
(1,0)\end{array}$ & $\begin{array}{l}74,0 \mathrm{~b} \\
(1,0)\end{array}$ & $\begin{array}{r}78,9 a \\
(2,1)\end{array}$ \\
\hline $\begin{array}{l}\text { Quantités de MS ingérées } \\
\left(\mathrm{g} / \mathrm{kg} \mathrm{P}^{0.75)}\right.\end{array}$ & $\begin{array}{l}87,2 \mathrm{a} \\
(8,9)\end{array}$ & $\begin{array}{l}48,3 \mathrm{~b} \\
(2,3)\end{array}$ & $\begin{array}{r}55,2 b \\
(10,0)\end{array}$ & $\begin{array}{l}48,6 b \\
(7,3)\end{array}$ & $\begin{array}{r}116,1 \mathrm{a} \\
(4,1)\end{array}$ & $\begin{array}{l}57,5 b \\
(12,2)\end{array}$ & $\begin{array}{l}67,4 \mathrm{c} \\
(3,3)\end{array}$ & $\begin{array}{r}61,6 \mathrm{~b} \\
(7,8)\end{array}$ \\
\hline $\begin{array}{l}\text { Durée journalière d'ingestion } \\
\text { (min/jour) }\end{array}$ & $\begin{array}{l}446 a \\
(38)\end{array}$ & $\begin{array}{l}264 b \\
(38)\end{array}$ & $\begin{array}{l}273 b \\
(36)\end{array}$ & $\begin{array}{l}347 \mathrm{c} \\
(54)\end{array}$ & $\begin{array}{l}360 a \\
(55)\end{array}$ & $\begin{array}{r}277 b \\
(17)\end{array}$ & $\begin{array}{l}190 \mathrm{c} \\
(53)\end{array}$ & $\begin{array}{r}272 b \\
(88)\end{array}$ \\
\hline $\begin{array}{l}\text { Durée unitaire d'ingestion } \\
\left(\mathrm{min} / \mathrm{g} / \mathrm{kg} \mathrm{P}^{0,75)}\right.\end{array}$ & $\begin{array}{r}5,11 \mathrm{a} \\
(0,77)\end{array}$ & $\begin{array}{r}5,47 a \\
(0,83)\end{array}$ & $\begin{array}{c}4,95 \mathrm{a} \\
(0,40)\end{array}$ & $\begin{array}{r}7,14 \mathrm{~b} \\
(1,84)\end{array}$ & $\begin{array}{l}3,10 \mathrm{ab} \\
(0,59)\end{array}$ & $\begin{array}{l}4,81 \mathrm{c} \\
(2,15)\end{array}$ & $\begin{array}{l}2,82 \mathrm{a} \\
(0,71)\end{array}$ & $\begin{array}{l}4,42 b \\
(1,25)\end{array}$ \\
\hline $\begin{array}{l}\text { Durée des grands repas } \\
\text { (min) }\end{array}$ & $\begin{array}{l}131 \mathrm{a} \\
(16)\end{array}$ & $\begin{array}{l}93 \mathrm{~b} \\
(15)\end{array}$ & $\begin{array}{l}87 \mathrm{~b} \\
(12)\end{array}$ & $\begin{array}{l}92 b \\
(12)\end{array}$ & $\begin{array}{l}76 a \\
(22)\end{array}$ & $\begin{array}{l}58 \mathrm{ac} \\
(17)\end{array}$ & $\begin{array}{l}34 b \\
(10)\end{array}$ & $\begin{array}{l}47 \mathrm{bc} \\
\text { (15) }\end{array}$ \\
\hline Nombre de repas par jour & $\begin{array}{r}7,1 \mathrm{a} \\
(1,2)\end{array}$ & $\begin{array}{l}5,0 \mathrm{~b} \\
(1,1)\end{array}$ & $\begin{array}{l}5,9 b \\
(1,3)\end{array}$ & $\begin{array}{l}8,5 \mathrm{c} \\
(0,9)\end{array}$ & $\begin{array}{l}10,4 a \\
(1,3)\end{array}$ & $\begin{array}{l}10,3 a \\
(2,5)\end{array}$ & $\begin{array}{l}8,7 \mathrm{a} \\
(3,1)\end{array}$ & $\begin{array}{r}10,1 \mathrm{a} \\
(1,2)\end{array}$ \\
\hline $\begin{array}{l}\text { Durée journalière } \\
\text { de rumination ( } \mathrm{min} / \text { jour) }\end{array}$ & $\begin{array}{l}527 a \\
(37)\end{array}$ & $\begin{array}{l}417 \mathrm{~b} \\
(91)\end{array}$ & $\begin{array}{l}548 a \\
(93)\end{array}$ & $\begin{array}{l}525 a \\
(60)\end{array}$ & $\begin{array}{l}580 a \\
(50)\end{array}$ & $\begin{array}{l}395 b \\
(44)\end{array}$ & $\begin{array}{l}470 \mathrm{c} \\
(62)\end{array}$ & $\begin{array}{c}329 \mathrm{bc} \\
(53)\end{array}$ \\
\hline $\begin{array}{l}\text { Durée unitaire de rumination } \\
\left(\mathrm{min} / \mathrm{g} / \mathrm{kg} \mathrm{P}^{0.75)}\right.\end{array}$ & $\begin{array}{r}6,04 a \\
(0,43)\end{array}$ & $\begin{array}{c}8,63 b \\
(1,70)\end{array}$ & $\begin{array}{r}9,93 \mathrm{c} \\
(1,08)\end{array}$ & $\begin{array}{l}10,80 \mathrm{c} \\
(1,37)\end{array}$ & $\begin{array}{r}4,99 \mathrm{a} \\
(0,41\rangle\end{array}$ & $\begin{array}{l}6,87 \mathrm{~b} \\
(2,15)\end{array}$ & $\begin{array}{l}6,97 \mathrm{~b} \\
(1,07)\end{array}$ & $\begin{array}{l}7,13 b \\
(0,37)\end{array}$ \\
\hline $\begin{array}{l}\text { Durée journalière } \\
\text { de mastication ( } \mathrm{min} / \text { jour) }\end{array}$ & $\begin{array}{l}973 a \\
(48)\end{array}$ & $\begin{array}{l}681 b \\
\langle 91\rangle\end{array}$ & $\begin{array}{l}821 c \\
(121)\end{array}$ & $\begin{array}{l}872 c \\
(35)\end{array}$ & $\begin{array}{l}940 \mathrm{a} \\
(78)\end{array}$ & $\begin{array}{l}672 b \\
(60\rangle\end{array}$ & $\begin{array}{l}660 \mathrm{~b} \\
(101)\end{array}$ & $\begin{array}{l}711 b \\
(136)\end{array}$ \\
\hline $\begin{array}{l}\text { Durée unitaire de mastication } \\
\left(\mathrm{min} / \mathrm{g} / \mathrm{kg} \mathrm{P} \mathrm{P}^{0.75)}\right.\end{array}$ & $\begin{array}{l}11,15 a \\
(1,09)\end{array}$ & $\begin{array}{l}14,10 \mathrm{~b} \\
(1,41)\end{array}$ & $\begin{array}{l}14,86 \mathrm{~b} \\
(1,20)\end{array}$ & $\begin{array}{l}17,94 \mathrm{c} \\
(2,45)\end{array}$ & $\begin{array}{r}8,09 a \\
(0,83)\end{array}$ & $\begin{array}{l}11,68 \mathrm{~b} \\
(3,41)\end{array}$ & $\begin{array}{c}9,79 a c \\
(1,49)\end{array}$ & $\begin{array}{r}11,55 b \\
(1,57)\end{array}$ \\
\hline $\begin{array}{l}\text { Temps de latence entre la fin } \\
\text { des grands repas et le début } \\
\text { de la rumination (min) }\end{array}$ & $\begin{array}{l}7 a \\
(8)\end{array}$ & $\begin{array}{c}42 \mathrm{bd} \\
\text { (19) }\end{array}$ & $\begin{array}{l}28 \mathrm{bd} \\
(14)\end{array}$ & $\begin{array}{l}20 \mathrm{ac} \\
(18)\end{array}$ & $\begin{array}{l}18 a \\
(5)\end{array}$ & $\begin{array}{l}66 \mathrm{~b} \\
(30)\end{array}$ & $\begin{array}{l}50 \mathrm{~b} \\
(27)\end{array}$ & $\begin{array}{l}49 \mathrm{~b} \\
(34)\end{array}$ \\
\hline
\end{tabular}

Seuil de signification $: P<0,05$ pour des lettres différentes.

Si on compare nos observations à celles de Geoffroy (1974), Thomas et Campling (1977), Carle et Dulphy (1980) et Focant (1984), on constate que les résultats des comparaisons entre espèces sont parfois divergents. L'individu (sexe, besoins), le mode de conduite et le type de régime jouent en effet un rôle tel que les comparaisons inter-espèces demeurent délicates.

Carle B., Dulphy J. P., 1980. Comportement alimentaire comparé des ovins et des bovins. Relation avec la digestion des aliments. Reprod. Nutr. Dévelop., 20, 1633-1639.

Dulphy J. P., Michalet-Doreau B., Demarquilly C., 1984. Etude comparée des quantités ingérées et du comportement alimentaire et mérycique d'ovins et de bovins recevant des ensilages d'herbes réalisés selon différentes techniques. Ann. Zootech., 33, 291-320.

Focant M., 1984. Comportement alimentaire, rumination, fermentations réticulo-ruminales et acides gras volatils plasmatiques, comparés chez la chèvre et le mouton ; influence du régime. Reprod. Nutr. Dévelop., 24, 239-250.

Geoffroy F., 1974. Etude comparée du comportement alimentaire et mérycique de deux petits ruminants : la chèvre et le mouton. Ann. Zootech., 23, 63-73.

Thomas S., Campling R. C., 1977. Comparisons of some factors affecting digestibility in sheep and cows. J. brit. Grass/d. Soc., 32, 33-41. 